\title{
EFFECTIVE RESULTS FOR RESTRICTED RATIONAL APPROXIMATION TO QUADRATIC IRRATIONALS
}

\author{
MICHAEL A. BENNETT AND YANN BUGEAUD \\ Dedicated, with admiration, to Andrzej Schinzel on the occasion of his 75th birthday
}

\begin{abstract}
In this paper, we deduce a number of effective lower bounds upon the distance to an integer of quantities of the shape $b^{n} \xi$, where $b$ and $n$ are integers and $\xi$ is a real quadratic irrational. For certain $\xi$, we obtain inequalities that approach in strength the corresponding ineffective results arising from the $p$-adic version of Roth's theorem.
\end{abstract}

\section{INTRODUCTION AND RESULTS}

Throughout the present note, we will denote by $b \geq 2$ an integer, $\mathcal{P}$ the set of prime factors of $b$, and $\xi$ a real algebraic number of degree $d \geq 2$. We are interested in studying combinatorial properties of the $b$-ary expansion of $\xi$.

By Liouville's inequality, there exists an effectively computable constant $c(\xi)$ such that

$$
\|q \xi\|>c(\xi) q^{-d+1},
$$

for $q \geq 1$, where $\|x\|$ denotes the distance from a real number $x$ to the nearest integer. Setting $q=b^{n}$ for $n \geq 1$, this implies in particular that the $n$-th decimal digit of $\xi$ cannot be followed by a block of $(d-1) n+\lceil(\log c(\xi)) /(\log 10)\rceil$ digits 0 .

A much stronger result is a consequence of Ridout's theorem [14]. Indeed, for every positive real number $\epsilon$, there exists a positive constant $c(\xi, \mathcal{P}, \epsilon)$ such that

$$
\left\|b^{n} \xi\right\|>c(\xi, \mathcal{P}, \epsilon) b^{-\epsilon n},
$$

for $n \geq 1$. This implies that, for every positive real number $\epsilon$ and for $n$ sufficiently large in terms of $\epsilon$, the $n$-th decimal digit of $\xi$ cannot be followed by a block of $\lceil\epsilon n\rceil$ digits 0 . It is a notorious fact that the constant $c(\xi, \mathcal{P}, \epsilon)$ is ineffective, in that it is not possible to find an explicit value for it from the proof in [14].

When $d \geq 3$, an effective strengthening of (1.1) was obtained by Feldman [12], by means of a sharpening [11] of Baker's theory of linear forms in (at least three) logarithms [1]. He established that

$$
\|q \xi\|>c(\xi) q^{-d+1+\tau(\xi)},
$$

for $q \geq 1$, where $c(\xi)$ and $\tau(\xi)$ are effectively computable positive numbers. The key argument in the proof of (1.3) is the introduction of the so-called parameter $b^{\prime}$ (see (2.1)) in the estimates for linear forms in logarithms (see also [4], where it is shown that estimates for linear forms in two logarithms, e.g. those obtained in

Date: August 26, 2011.

2010 Mathematics Subject Classification. Primary: 11J68, Secondary: 11A63, 11D61, 11J86. 
[13], are sufficient to obtain (1.3)). An alternative approach has been developed by Bombieri [5].

It is convenient to restate the above results in terms of the exponents of approximation $v_{b}$ and $v_{b}^{\text {eff }}$. We denote by $v_{b}(\xi)$ the infimum of the real numbers $v$ for which the inequality

$$
\left\|b^{n} \xi\right\|>\left(b^{n}\right)^{-v}
$$

holds for every sufficiently large positive integer $n$. Likewise, $v_{b}^{\text {eff }}(\xi)$ denotes the infimum of the real numbers $v$ for which there exists an effectively computable constant $c(\xi, v)$ such that

$$
\left\|b^{n} \xi\right\|>c(\xi, v)\left(b^{n}\right)^{-v},
$$

for $n \geq 1$. In view of (1.2) and (1.3), we have $v_{b}(\xi)=0$ and, assuming that $\xi$ is of degree at least three,

$$
v_{b}^{\mathrm{eff}}(\xi) \leq d-1-\tau(\xi),
$$

for some effectively computable positive real number $\tau(\xi)$. As noted previously, the values $v_{b}(\xi)$ and $v_{b}^{\text {eff }}(\xi)$ provide information on the lengths of blocks of the digit 0 (and of the digit $b-1$ ) in the $b$-ary expansion of $\xi$.

It only remains for us to consider the case when $\xi$ is of degree two. In this situation, the first effective estimates for $\left\|b^{n} \xi\right\|$ were given by Schinzel [15] in a landmark paper published in 1967, in which he developed the theory of linear forms in two $p$-adic logarithms, making completely explicit the bounds of Gelfond.

Theorem 1.1. (Schinzel) For every integer $b \geq 2$ and every quadratic real number $\xi$, we have

$$
\left\|b^{n} \xi\right\|>b^{-n} \exp \left\{c(\xi, b) n^{1 / 7}\right\},
$$

for $n \geq 1$, where $c(\xi, b)$ is a positive effectively computable constant depending only on $\xi$ and $b$.

Since the publication of Schinzel's paper, there have been important improvements in the theory of linear forms in two $p$-adic logarithms. It is now possible to improve Theorem 1.1 as follows.

Theorem 1.2. For every integer $b \geq 2$ and every quadratic real number $\xi$, we have

$$
\left\|b^{n} \xi\right\|>c(\xi, b) b^{-(1-\tau(\xi, \mathcal{P})) n},
$$

for $n \geq 1$, where $c(\xi, b)$ and $\tau(\xi, \mathcal{P})$ are positive effectively computable constants depending only on $\xi$ and $b$, and on $\xi$ and on the set $\mathcal{P}$ of prime factors of $b$, respectively. In particular, we have

$$
v_{b}^{\text {eff }}(\xi) \leq 1-\tau(\xi, \mathcal{P}) .
$$

We obtain in (1.5) an improvement upon (1.1) of a similar strength as (1.4). Again, the source of the strengthening is the introduction of the parameter $b^{\prime}$ (see (2.1)) in the estimates for linear forms in non-Archimedean logarithms.

We point out that the numerical constant occurring in (1.5) depends on the set of prime divisors of $b$, while in Schinzel's theorem the dependence is in terms of $b$.

The effective improvement of Liouville's inequality in (1.4) is fully independent of the base $b$. While this is not the case in Theorem 1.2, the theory of linear forms in logarithms enables us to establish the following 'uniform' result. 
Theorem 1.3. Let $p$ be a prime number. There exists an effectively computable absolute positive constant $\tau_{1}$ such that

$$
v_{p}^{\mathrm{eff}}\left(\sqrt{p^{2}+1}\right) \leq 1-\tau_{1} .
$$

Further progress on estimates for linear forms in two non-Archimedean logarithms enables us to somewhat generalize (1.6). In particular, one can deduce the existence of an effectively computable absolute positive constant $\tau_{2}$ such that

$$
v_{b}^{\text {eff }}\left(\sqrt{b^{2 k}+1}\right) \leq 1-\tau_{2},
$$

for every positive integer $k$ and every $b \geq 2$; we explain this at the end of Section 2 .

As is often the case when applicable, the theory of Padé approximants leads to sharper bounds and, in particular, considerable effective improvements of Liouville's inequality. The main result of the present note shows that, for a special class of quadratic numbers, the exponent $v_{b}^{\text {eff }}$ can be proved to be very small.

Theorem 1.4. For every integers $b \geq 2$ and $k \geq 1$, we have

$$
v_{b}^{\text {eff }}\left(\sqrt{b^{2 k}+1}\right) \leq(\log 48) /(k \log b) .
$$

This theorem shows that, for every integer $b \geq 2$, there exist arbitrarily large quadratic real numbers $\xi$ such that

$$
v_{b}^{\text {eff }}(\xi) \leq 9 /(2 \log \xi) .
$$

In particular, for every positive real number $\epsilon$, there exist quadratic real numbers $\xi$ such that $v_{b}^{\text {eff }}(\xi)<\epsilon$.

Theorem 1.2 is not entirely satisfactory since the right-hand side of (1.5) is dependent upon the base $b$, in contrast to the situation in (1.4). This suggests the following

Open problem. To prove that for every quadratic number $\xi$ there exists an effectively computable positive number $\tau(\xi)$ such that

$$
v_{b}^{\mathrm{eff}}(\xi) \leq 1-\tau(\xi)
$$

for every $b \geq 2$.

Remark 1. The effective results on the number of digit changes in the $b$-ary expansion of algebraic irrational numbers $\xi$ obtained in [8] tend to suggest that $v_{b}^{\text {eff }}(\xi)$ is small, but do not actually provide explicit evidence in this regard.

Remark 2. The method of proof of Theorem 1.4 is flexible enough to yield analogous results for certain more general families of quadratic numbers.

\section{Proofs of Theorems 1.2 AND 1.3 : LineAR FORMS IN LOGARIThMS}

We state below a slightly simplified proof of Théorème 3 of [10]. This is the key tool for the proofs of Theorems 1.2 and 1.3.

Let $p$ be a prime number and denote by $\overline{\mathbb{Q}}_{p}$ an algebraic closure of the $p$-adic field $\mathbb{Q}_{p}$. We equip the field $\overline{\mathbb{Q}}_{p}$ with the ultrametric absolute value $|x|_{p}=p^{-\nu_{p}(x)}$, where $\nu_{p}$ denotes the unique extension to $\overline{\mathbb{Q}}_{p}$ of the standard $p$-adic valuation over $\mathbb{Q}_{p}$ normalized by $\nu_{p}(p)=1$ (we set $\nu_{p}(0)=+\infty$ ). Let $\alpha_{1}$ and $\alpha_{2}$ be algebraic over 
$\mathbb{Q}$ and regard them as elements of the field $\overline{\mathbb{Q}}_{p}$. Write $D=\left[\mathbb{Q}\left(\alpha_{1}, \alpha_{2}\right)\right.$ : $\left.\mathbb{Q}\right]$. Our goal is to deduce a lower bound for the ultrametric absolute value of

$$
\Lambda=\alpha_{1}^{b_{1}}-\alpha_{2}^{b_{2}}
$$

where $b_{1}$ and $b_{2}$ are arbitrary positive rational integers.

We assume that $\nu_{p}\left(\alpha_{1}\right)=\nu_{p}\left(\alpha_{2}\right)=0$ and denote by $g$ the smallest positive integer such that

It is easily seen that

$$
\nu_{p}\left(\alpha_{1}^{g}-1\right)>0 \quad \text { and } \quad \nu_{p}\left(\alpha_{2}^{g}-1\right)>0 .
$$

$$
g \leq p^{D}-1 .
$$

Let $A_{1}>1, A_{2}>1$ be real numbers such that

$$
\log A_{i} \geq \max \left\{h\left(\alpha_{i}\right), \frac{\log p}{D}\right\}, \text { for } i=1 \text { and } 2,
$$

where $h$ denotes the logarithmic Weil height. Set

$$
b^{\prime}=\frac{b_{1}}{D \log A_{2}}+\frac{b_{2}}{D \log A_{1}} .
$$

As a historical note, we should mention that in the early estimates for linear forms in logarithms, the term $b^{\prime}$ was replaced by the larger quantity $\max \left\{b_{1}, b_{2}, 3\right\}$.

Theorem 2.1. With the above notation, if the algebraic numbers $\alpha_{1}$ and $\alpha_{2}$ are multiplicatively independent, then $\nu_{p}(\Lambda)$ is bounded above by

$$
\frac{24 p g}{(p-1)(\log p)^{4}} D^{4}\left(\max \left\{\log b^{\prime}+\log \log p+0.4, \frac{10 \log p}{D}, 10\right\}\right)^{2} \log A_{1} \log A_{2} \text {. }
$$

To proceed with the proof of Theorem 1.2, we simply reproduce Schinzel's argument, replacing the use of his estimates for linear forms in two $p$-adic logarithms by Theorem 2.1. Without loss of generality, we assume that $\xi$ is the positive root of the non-square positive integer $a$. We shall keep this notation in the sequel to this section.

Proof of Theorem 1.2. Let $x$ be a positive integer and write

$$
x^{2}-a b^{2 n}=\left(x+\xi b^{n}\right) \cdot\left(x-\xi b^{n}\right)=: \Delta .
$$

Suppose that $\eta$ is the fundamental unit of the quadratic field generated by $\sqrt{a}$. We use the sign $\div$ to denote Galois conjugacy in $\mathbb{Q}(\sqrt{a})$. Write

$$
x-\xi b^{n}=\delta \eta^{m},
$$

where $\delta$ and $m$ are such that

$$
|\Delta \eta|^{1 / 2} \cdot \eta^{-1}<|\delta| \leq|\Delta \eta|^{1 / 2}, \quad \delta \bar{\delta}=\Delta .
$$

Observe that

$$
\left(x^{2}-\xi^{2} b^{2 n}\right)-\left(x-\xi b^{n}\right)^{2}=\Delta-\delta^{2} \eta^{2 m}=2\left(\xi b^{n} x+\xi^{2} b^{2 n}\right) .
$$

Let us factor $b=p_{1}^{\ell_{1}} \cdots p_{s}^{\ell_{s}}$ as a product of powers of distinct prime numbers, choose $j$ so that $\ell_{j}=\max \left\{\ell_{1}, \ldots, \ell_{s}\right\}$, and put $p=p_{j}$ and $\ell=\ell_{j}$.

We wish to bound $\nu_{p}\left(\Delta-\delta^{2} \eta^{2 m}\right)=\nu_{p}\left(\bar{\delta} / \delta-\eta^{2 m}\right)$. Observe that the logarithmic Weil height of $\bar{\delta} / \delta$ is $\ll \log \Delta$ (here and henceforth, the constant implied by $\ll$ depends at most on $a$ ). Using Theorem 2.1, it follows that

$$
\nu_{p}\left(\bar{\delta} / \delta-\eta^{2 m}\right) \ll p^{2}(\log \Delta) \log ^{2}(m /(\log \Delta)) .
$$


On the other hand, we derive from (2.2) that

$$
\nu_{p}\left(\bar{\delta} / \delta-\eta^{2 m}\right) \geq n \ell,
$$

whereby our choice of $\delta$ and $m$ yields

$$
m \ll n \text {. }
$$

We thus have

$$
n \ell \ll p^{2}(\log \Delta) \log ^{2}(n /(\log \Delta)),
$$

whence

$$
n \ll\left(\frac{p^{2}}{\ell} \log ^{2} \frac{p}{\ell}\right)(\log \Delta)
$$

and

$$
\frac{\log \Delta}{\log b} \gg \frac{n}{p^{2} \log ^{2} p} \cdot \frac{\ell}{\ell_{1} \log p_{1}+\ldots+\ell_{s} \log p_{s}} \gg \frac{n}{p^{2}\left(\log ^{2} p\right)\left(\log p_{1} \cdots p_{s}\right)} .
$$

Choosing $x$ to be the nearest integer to $\sqrt{a} b^{n}$, this proves that there exists an effectively computable positive constant $\kappa$ depending only on $p_{1}, \ldots, p_{s}$ and $a$ such that

$$
\left\|b^{n} \sqrt{a}\right\|=\left|x-\xi b^{n}\right| \geq\left(2 \xi b^{n}+1\right)^{-1} \cdot b^{\kappa n} .
$$

This concludes the proof of Theorem 1.2.

An annoying feature of estimates for linear forms in $p$-adic logarithms is the strong dependence of the bound on the prime number $p$, unless the algebraic numbers involved are $p$-adically close to 1 . This is expressed in Theorem 2.1 by means of the parameter $g$. Actually, one obtains a stronger result for certain special numbers $\xi$. Let $b \geq 2$ be an integer and, for $k \geq 1$, set

$$
a_{k}=b^{2 k}+1 \text {. }
$$

Then

$$
\eta_{k}:=b^{k}+\sqrt{a_{k}}
$$

is the fundamental unit (of norm -1 ) in $\mathbb{Q}\left(\sqrt{a_{k}}\right)$. Observe that $\eta_{k}$ is congruent to 1 modulo $p^{2 k \ell}$, where $\ell$ is such that $p^{\ell}$ divides $b$ but $p^{\ell+1}$ does not. When $p$ divides $b$, this shows that Theorem 2.1 applies with $g=1$. In this case, this yields an inequality of the shape

$$
\left\|b^{n} \sqrt{a_{k}}\right\|>c(b, k) b^{-n} \cdot p^{\tau_{3} n},
$$

for an effectively computable absolute positive constant $\tau_{3}$ and an effectively computable positive constant $c(b, k)$.

For the particular case $b=p$ and $k=1$, we have

$$
\left\|p^{n} \sqrt{p^{2}+1}\right\|>c(p) p^{-\left(1-\tau_{4}\right) n},
$$

for an effectively computable absolute positive constant $\tau_{4}$ and an effectively computable positive constant $c(p)$. This proves Theorem 1.3.

A further refinement of estimates for linear forms in two $p$-adic logarithms appeared in [6]. A new parameter was introduced, which proves useful when the two algebraic numbers involved are both congruent to 1 modulo a large power of the prime $p$. We are in precisely this situation for numbers of the shape $\sqrt{p^{2 k}+1}$; in 
this case, we deduce the existence of an effectively computable absolute positive constant $\tau_{5}$ such that

$$
\left\|p^{n} \sqrt{p^{2 k}+1}\right\|>p^{-\left(1-\tau_{5}\right) n}
$$

for every integer $n$ exceeding some effectively computable number depending on $p$ and $k$. Note that, in [6], the main corollaries rely upon the assumption that the algebraic numbers $\alpha_{1}$ and $\alpha_{2}$ involved are in fact rational. While this is not the case in the present situation, we can still deduce (2.3) from the rather less "userfriendly" Theorem 1 of [6]. A further generalization of the results of [6] to $b$-adic logarithms was obtained in [7], again under the assumption that the arguments of the logarithms $\alpha_{1}$ and $\alpha_{2}$ are rational numbers. The techniques of [7], however, yield similar estimates when both $\alpha_{i}$ are more general algebraic numbers (provided that, of course, the analogues of hypotheses (H1) and (H2) are satisfied). Ultimately, this allows us to show the existence of an effectively computable absolute positive constant $\tau_{6}$ such that

$$
\left\|b^{n} \sqrt{b^{2 k}+1}\right\|>b^{-\left(1-\tau_{6}\right) n}
$$

for every integer $n$ exceeding some effectively computable number depending on $b$ and $k$.

In many questions where one may employ the theory of linear forms in logarithms of algebraic numbers close to 1 , it transpires that the hypergeometric method is equally applicable and often provides spectacular improvements (see e.g. most of the problems considered in the survey [9]). This is the case in the present situation, as we shall see in the next section.

\section{Proof of Theorem 1.4 : Padé approximants to $(1-z)^{1 / 2}$}

In this section, we will prove Theorem 1.4. This result can also be obtained from careful analysis of the proof of Theorem 1.3 of [2]; the argument we provide here is a rather simplified version.

Let us define

$$
I_{n_{1}, n_{2}}(x)=\frac{1}{2 \pi i} \int_{\gamma} \frac{(1-z x)^{n_{2}}(1-z x)^{1 / 2}}{z^{n_{1}+1}(1-z)^{n_{2}+1}} d z
$$

for $n_{1}$ and $n_{2}$ positive integers, $\gamma$ a closed, counter-clockwise contour enclosing $z=0$ and $z=1$, and $|x|<1$. Cauchy's theorem readily implies that

$$
I_{n_{1}, n_{2}}(x)=P_{n_{1}, n_{2}}(x)-(1-x)^{1 / 2} Q_{n_{1}, n_{2}}(x)
$$

where $P_{n_{1}, n_{2}}(x)$ and $Q_{n_{1}, n_{2}}(x)$ are polynomials with rational coefficients and degrees $n_{1}$ and $n_{2}$, respectively. These are, in fact, Padé approximants to $(1-z)^{1 / 2}$, though we will not explicitly use this fact. Calculating the relevant residues, we have

$$
P_{n_{1}, n_{2}}(x)=\sum_{k=0}^{n_{1}}\left(\begin{array}{c}
n_{2}+1 / 2 \\
k
\end{array}\right)\left(\begin{array}{c}
n_{1}+n_{2}-k \\
n_{2}
\end{array}\right)(-x)^{k}
$$

and

$$
Q_{n_{1}, n_{2}}(x)=\sum_{k=0}^{n_{2}}\left(\begin{array}{c}
n_{1}-1 / 2 \\
k
\end{array}\right)\left(\begin{array}{c}
n_{1}+n_{2}-k \\
n_{1}
\end{array}\right)(-x)^{k} .
$$

In particular, if $n_{1} \geq n_{2}$, since

$$
\left(\begin{array}{c}
n+1 / 2 \\
k
\end{array}\right) 4^{k} \in \mathbb{Z}
$$


for $n \geq 1$, we have that

$$
4^{n_{1}} P_{n_{1}, n_{2}}(x), 4^{n_{1}} Q_{n_{1}, n_{2}}(x) \in \mathbb{Z}[x] .
$$

We proceed as follows. For fixed positive integers $b, n$ and $k$, with $b>1$, we define $\epsilon$ and an integer $m$ such that

$$
\epsilon=\sqrt{b^{2 k}+1} \cdot b^{n}-m \text { and }|\epsilon|=\left\|b^{n} \sqrt{b^{2 k}+1}\right\| .
$$

Our goal, thus, is to derive a lower bound upon $|\epsilon|$. Since $|\epsilon|<1 / 2$, we have that $m<\sqrt{b^{2 k}+1} \cdot b^{n}+1 / 2$. Let us define

$$
\alpha=1+\frac{k \log b}{\log 2} \geq 2,
$$

and suppose that

$$
n_{2}=\left[\frac{n \log 2}{2 k^{2} \log b}+\frac{\log 2}{2 k \log b}\right]+1 \text { and } n_{1}=\left[\alpha n_{2}\right]+\delta+1,
$$

where $\delta$ is one of 0 or 1 . Assume further that

$$
n>\frac{9 k^{3} \log ^{2} b}{\log ^{2} 2} .
$$

Note that

$$
n_{1}-n_{2}>(\alpha-1) n_{2}=\frac{k n_{2} \log b}{\log 2}>\frac{n}{2 k}+\frac{1}{2}
$$

and so, if we define $\tau=2 k\left(n_{1}-n_{2}\right)-n-k$, then $\tau>0$. Substituting $x=-1 / b^{2 k}$ into (3.1) and defining

$$
P=4^{n_{1}} b^{2 k n_{1}} P_{n_{1}, n_{2}}\left(-1 / b^{2 k}\right), Q=4^{n_{1}} b^{2 k n_{2}+\tau} Q_{n_{1}, n_{2}}\left(-1 / b^{2 k}\right)
$$

and

$$
I=4^{n_{1}} b^{2 k n_{1}} I_{n_{1}, n_{2}}\left(-1 / b^{2 k}\right),
$$

it follows that $P$ and $Q$ are integers, with

$$
P-\sqrt{b^{2 k}+1} \cdot b^{n} Q=I .
$$

We therefore have

$$
\sqrt{b^{2 k}+1}(\epsilon P+I m)=\left(b^{2 k}+1\right) b^{n}(P-Q m) .
$$

Arguing as in Lemma 4 of [3], for at least one choice of $\delta \in\{0,1\}$ in (3.2) we necessarily have that $P \neq Q m$, whereby

$$
|\epsilon| \cdot|P| \geq \sqrt{b^{2 k}+1} b^{n}-|I| \cdot|m| .
$$

Since $m<\sqrt{b^{2 k}+1} b^{n}+\frac{1}{2}<\frac{3}{2} \sqrt{b^{2 k}+1} b^{n}$, if we can show that $|I|<1 / 3$, then we may conclude that

$$
|\epsilon|>\frac{1}{2} \cdot \sqrt{b^{2 k}+1} \cdot b^{n} \cdot|P|^{-1} .
$$

We now proceed to find an upper bound upon $|I|$. Arguing as in [2], we have

$$
\left|I_{n_{1}, n_{2}}(x)\right|=\frac{|x|^{n_{1}+n_{2}+1}}{\pi} \int_{0}^{1} \frac{v^{n_{2}+1 / 2}(1-v)^{n_{1}-1 / 2} d v}{(1-(1-v) x)^{n_{2}+1}}
$$

and so

$$
|I|=4^{n_{1}} b^{2 k n_{1}}\left|I_{n_{1}, n_{2}}\left(-1 / b^{2 k}\right)\right|<4^{n_{1}} b^{-2 k\left(n_{2}+1\right)} \pi^{-1} \int_{0}^{1} v^{n_{2}+1 / 2}(1-v)^{n_{1}-1 / 2} d v .
$$


If $b^{k}=2$, then $k=1, b=2, \alpha=2, n_{2}=\left[\frac{n+3}{2}\right], n_{1}=2 n_{2}+\delta+1$ and so

$$
|I|<4^{n_{2}+1} \pi^{-1}(4 / 27)^{n_{2}+\frac{1}{2}}<1 / 3 .
$$

Otherwise,

$$
|I|<4^{n_{1}} b^{-2 k\left(n_{2}+1\right)} \pi^{-1} \int_{0}^{1} v^{n_{2}-\frac{1}{2 \alpha}}(1-v)^{\alpha\left(n_{2}-\frac{1}{2 \alpha}\right)} d v
$$

whereby

$$
|I|<4^{n_{1}} b^{-2 k\left(n_{2}+1\right)} \pi^{-1}\left(\frac{\alpha^{\alpha}}{(1+\alpha)^{1+\alpha}}\right)^{n_{2}-\frac{1}{2 \alpha}}<4^{\alpha n_{2}+2} b^{-2 k\left(n_{2}+1\right)}(2 \alpha)^{-n_{2}} .
$$

Since (3.2) and (3.3) together imply that $n_{2} \geq 10$, it follows from our choice of $\alpha$ that

$$
|I|<4^{n_{2}+2} b^{-2 k}(2 \alpha)^{-n_{2}}<1 / 3,
$$

as desired, where the last inequality is a consequence of $b^{k} \geq 3$.

To conclude, we will combine inequality (3.4) with an upper bound for $|P|$. If $0<r<1$, then

$$
P_{n_{1}, n_{2}}(x)=\frac{1}{2 \pi i} \int_{\Gamma} \frac{(1-z x)^{n_{2}}(1-z x)^{1 / 2}}{z^{n_{1}+1}(1-z)^{n_{2}+1}} d z
$$

where $\Gamma$ is defined by $|z|=r$, oriented positively. Writing $z=r e^{i \theta}$, we have

$$
\left|P_{n_{1}, n_{2}}(x)\right| \leq \frac{1}{2 \pi} \int_{0}^{2 \pi}\left|\frac{(1-z x)^{n_{2}}(1-z x)^{1 / 2}}{z^{n_{1}+1}(1-z)^{n_{2}+1}}\right| d \theta
$$

and so

$$
\left|P_{n_{1}, n_{2}}(x)\right| \leq \frac{1}{r^{n_{1}+1}} \max _{0 \leq \theta \leq 2 \pi}\left|\frac{\left(1-r e^{i \theta} x\right)^{n_{2}+1 / 2}}{\left(1-r e^{i \theta}\right)^{n_{2}+1}}\right| .
$$

Since $|x|<1$ and $0<r<1$, both $\left|1-r e^{i \theta}\right|$ and $\left|\frac{1-r e^{i \theta}}{1-r e^{i \theta} x}\right|$ are increasing functions of $\theta$ on the interval $[0, \pi]$ (and hence minimal at $\theta=0$ ), whereby

$$
\left|P_{n_{1}, n_{2}}(x)\right| \leq \frac{\sqrt{1-r x}}{r^{n_{1}+1}(1-r)}\left(\frac{1-r x}{1-r}\right)^{n_{2}} .
$$

Taking $x=-1 / b^{2 k}$ and $r=\alpha /(1+\alpha)$, using only that $b^{k} \geq 2$, (3.5) implies that

$$
|P|<5 \cdot 12^{n_{1}} b^{2 k n_{1}} .
$$

With (3.4), we thus have

$$
|\epsilon|>\frac{1}{10} \cdot b^{n+k-2 k n_{1}} \cdot 12^{-n_{1}} .
$$

Since (3.2) yields

$$
n_{1}<\frac{n}{2 k}\left(1+\frac{\log 2}{k \log b}\right)+\frac{k \log b}{\log 2}+4
$$

it follows that

where

$$
|\epsilon|>\frac{1}{10} \cdot b^{-\tau} \cdot 12^{-n_{1}}
$$

$$
\tau=\frac{n \log 2}{k \log b}+\frac{2 k^{2} \log b}{\log 2}+7 k .
$$


Applying (3.3), we may conclude that $\tau<\frac{2 n \log 2}{k \log b}$ and hence

$$
\left\|b^{n} \sqrt{b^{2 k}+1}\right\|>10^{-7} \cdot 48^{-n / k},
$$

which immediately implies Theorem 1.4.

\section{REFERENCES}

[1] A. Baker, Linear forms in the logarithms of algebraic numbers I-IV, Mathematika 13 (1966), 204-216; 14 (1967), 102-107 and 220-224; 15 (1968), 204-216.

[2] M. Bauer and M. Bennett, Application of the hypergeometric method to the generalized Ramanujan-Nagell equation, Ramanujan J. 6 (2002), 209-270.

[3] F. Beukers, On the generalized Ramanujan-Nagell equation I, Acta Arith. 38 (1980/81), 389-410.

[4] Yu. Bilu and Y. Bugeaud, Démonstration du théorème de Baker-Feldman via les formes linéaires en deux logarithmes, J. Théor. Nombres Bordeaux 12 (2000), 13-23.

[5] E. Bombieri, Effective Diophantine Approximation on $\mathbb{G}_{m}$, Ann. Scuola Norm. Sup. Pisa Cl. Sci. 20 (1993), 61-89.

[6] Y. Bugeaud, Linear forms in p-adic logarithms and the Diophantine equation $\left(x^{n}-1\right) /(x-1)=y^{q}$, Math. Proc. Cambridge Philos. Soc. 127 (1999), 373-381.

[7] Y. Bugeaud, Linear forms in two m-adic logarithms and applications to Diophantine problems, Compositio Math. 132 (2002), 137-158.

[8] Y. Bugeaud, On the b-ary expansion of an algebraic number, Rend. Sem. Mat. Univ. Padova 118 (2007), 217-233.

[9] Y. Bugeaud, Linear forms in the logarithms of algebraic numbers close to 1 and applications to Diophantine equations. In: Diophantine equations, 59-76, Tata Inst. Fund. Res. Stud. Math., 20, Tata Inst. Fund. Res., Mumbai, 2008.

[10] Y. Bugeaud and M. Laurent, Minoration effective de la distance p-adique entre puissances de nombres algébriques, J. Number Theory 61 (1996), 311-342.

[11] N.I. Feldman, Improved estimate for a linear form of the logarithms of algebraic numbers, Mat. Sb. 77 (1968), 256-270 (in Russian); English transl. in Math. USSR. Sb. 6 (1968) 393406.

[12] N.I. Feldman, An effective refinement of the exponent in Liouville's theorem, Iz. Akad. Nauk SSSR, Ser. Mat. 35 (1971), 973-990 (in Russian); English transl. in Math. USSR. Izv. 5 (1971) 985-1002.

[13] M. Laurent, M. Mignotte, and Yu. Nesterenko, Formes linéaires en deux logarithmes et déterminants d'interpolation, J. Number Theory 55 (1995), 285-321.

[14] D. Ridout, The p-adic generalization of the Thue-Siegel-Roth theorem, Mathematika 5 (1958), 40-48.

[15] A. Schinzel, On two theorems of Gel'fond and some of their applications, Acta Arith. 13 (1967), 177-236.

Michael A. Bennett, Department of Mathematics, University of British Columbia, Vancouver, British Columbia, V6T 1Z2, CANADA

E-mail address: bennett@math.ubc.ca

Yann Bugeaud, Mathématiques, Université de Strasbourg, 7, Rue René Descartes, 67084 Strasbourg, FRANCE

E-mail address: bugeaud@math.unistra.fr 